Published online 2017 April 13.

Abstract

\title{
A Survey of Knowledge and Attitude of Staff and Hospital Administrators in the Implementation of Archiving and Transmission of Medical Images (PACS) in Hospitals in Ahwaz
}

\author{
Fateme Panahi, ${ }^{1, *}$ Mehrdad Gholami, ${ }^{2}$ and Sera Mayahi \\ ${ }^{1}$ Lorestan University of Medical Sciences, Lorestan, Iran \\ ${ }^{2} \mathrm{MD}$ \\ "Corresponding author: Fateme Panahi, Lorestan University of Medical Sciences, Lorestan, Iran. E-mail: panahi.fateme.radiology71@gmail.com \\ Received 2016 December 21; Accepted 2017 February 08.
}

\begin{abstract}
Background: Medical image archiving and transmission system (PACS) are systems used to capture, store, transmit and display medical images and archive medical images used. Information is exchanged through an internal network in the health center or the Internet platform.

Objectives: The aim of this study was to analyze the degree of knowledge and attitude of staff and hospital administrators in the implementation of archiving and transmission of medical images (PACS) in hospitals in Ahwaz.

Methods: This study is cross-sectional study. The population under study were the staff and hospital administrators at the medical schools in Ahwaz. A total of 176 questionnaires were distributed. Data collection tool was a questionnaire with two parts: the first part of the questionnaire consisted of demographic information which had 10 multi choice questions about the knowledge and attitude of staff and hospital administrators in the implementation of archiving and transmission of medical images (PACS). And the second Data collection tool was a check list about assessing the technical and other potential obstacles.

Results: The average score of the knowledge of the users and administrators with PACS and related systems were65/30 and 5/27 respectively. The attitudes of users and administrators in implementing PACS system were2/76 and 5/59 respectively. According to the managers, the major obstacles for the implementation of PACS system were a lack of awareness of the benefits of PACS for both the users and administrators and cost.

Conclusions: According to the present study, the potential barriers to the implementation of PACS in hospitals is the lack of knowledge of the managers and employees of the benefits of PACS system (80\%). Before the implementation of PACS systems in hospitals it must be considered necessary background to increase knowledge of the workers and managers in this regard, short-term in-service training courses and providing educational pamphlets can be useful also.
\end{abstract}

Keywords: PACS, Awareness, Barriers to Implementation, Staff and Administrators

This is an abstract presented in the 33rd Iranian congress of radiology (ICR) and the 15th congress of Iranian radiographic science association (IRSA). 